\title{
A Numerical Study of Periodically Forced Flows Using a Spectral-Projection Method
}

\author{
J. M. Lopez ${ }^{1}$ and Jie Shen ${ }^{2}$ \\ ${ }^{1}$ Department of Mathematics, Arizona State University, Tempe, AZ 85287, USA \\ ${ }^{2}$ Department of Mathematics, The Pennsylvania State University, University Park, \\ PA 16802, USA \\ email: lopez@math.la.asu.edu; shen@math.psu.edu
}

Key Words: INCOMPRESSIBLE, SPECTRAL-GALERKIN, PROJECTION, TAYLORCOUETTE, PERIODICALLY FORCED FLOW

\begin{abstract}
Recent experiments (Weisberg, Kevrekidis \& Smits 1997) have demonstrated that the centrifugal instability leading to Taylor vortex flow can be controlled by harmonic oscillations of the inner cylinder in the axial direction. Marques \& Lopez (1997) used linear Floquet analysis to study the control of the instability for the flow between two infinite length co-axial cylinders. However, nonlinear endwall effects have not been investigated previously for the physically realistic case of finite length co-axial cylinders. In this paper, we use an accurate and efficient spectral-projection scheme for the nonlinear axisymmetric Navier-Stokes equations to examine the endwalls effects and the breaking of space-time symmetries.
\end{abstract}

\section{Problem description and the numerical method}

In $\mathrm{Hu} \&$ Kelly (1995) and Marques \& Lopez (1997) Floquet analysis was used to study the stability of the flow between two co-axial cylinders, the outer one being stationary and the inner one rotating at a fixed rate (the usual Taylor-Couette flow) as well as oscillating harmonically in the axial direction. This system has also been investigated experimentally by Weisberg et al. (1997). The inner cylinder oscillates in the axial direction with velocity $U \sin \Omega t$ and rotates at constant angular velocity $\Omega_{i}$. Its radius is $r_{i}$ and that of the outer stationary cylinder is $r_{o}$. The annular gap between them is $d=$ $r_{o}-r_{i}$. These parameters are combined to give the following nondimensional governing parameters: the radius ratio $e=r_{i} / r_{o}$, the Couette flow Reynolds number $R e_{i}=d r_{i} \Omega_{i} / \nu$, the axial Reynolds number $R e_{a}=d U / \nu$, and the nondimensional frequency $\omega_{f}=d^{2} \Omega / \nu$, where $\nu$ is the kinematic viscosity of the fluid.

The basic state consists of circular Couette flow with a superimposed annular Stokes flow. It is independent of the axial and azimuthal directions, and time-periodic with the period of the forcing; an analytic description of the basic flow is derived in Marques \& Lopez (1997). Over an extensive range of parameter space, the primary bifurcation is to an axisymmetric state that is periodic in the axial direction and time, with the same temporal period 
as the forcing (Weisberg et al. 1997; Marques \& Lopez 1997). The system also has a space-time symmetry, translation by half a period in time together with a reflection in $z$.

The analysis of Marques \& Lopez (1997), however, showed that in narrow windows of parameter space, where interaction and competition between different axial modes occurs, the primary bifurcation is to a state that is periodic in both the axial and the azimuthal directions, and temporally has the forcing frequency as well as a new frequency $\omega_{s}$, so that the dynamics are on a 2-torus. These regions in parameter space are pockets of spatio-temporal complexity, as the solutions are no longer axisymmetric $(n \neq 0)$. Normally, in the unforced Taylor-Couette flow, these azimuthal modes are interpreted as either single $(n=1)$ or double $(n=2)$ spirals, but here, they can manifest themselves as tilted, wobbling, and deforming Taylor cells, due to the interaction with the axial and temporal periodicities. Such tilted cells were noted in the experiments of Weisberg (1996) within the same parameter range, but were not investigated in detail in that study.

All the previous theoretical results discussed above correspond to the idealized case of infinite length cylinders, although the presence of endwalls is accounted for to leading order by imposing zero net mass flux via the pressure gradient (see Marques \& Lopez 1997). Further, the above considerations are only of the system at the point of instability. We now study the influence on the nonlinear dynamics due to the finite length of the system.

In order solve the nonlinear Navier-Stokes equations accurately at low cost, we employ a highly efficient and accurate spectral-projection method in which the time variable is discretized by using a second-order projection scheme and the spatial variables are discretized by using a spectralGalerkin method (cf. Shen 1994; Shen 1997). We refer to Lopez \& Shen (1998) for more details on the spectral-projection scheme for axisymmetric Navier-Stokes equations in enclosed cylinder(s).

The flow starts from rest and satisfies the following boundary conditions:

$$
\begin{aligned}
& u=0, v=R e_{i}, w=R e_{a} \sin \omega_{f} t \quad \text { at } r=r_{i} \\
& u=v=w=0 \quad \text { at } r=r_{o} \text { and } z=0, \Lambda
\end{aligned}
$$

This flow has discontinuous boundary conditions for $v$ and $w$, the azimuthal and axial components of velocity, at the corners where the inner cylinder meets the stationary endwalls. A regularized boundary layer function is used as in Lopez \& Shen (1998).

In all our computations, we have used $64 \times 80$ modes for all quantities, although $48 \times 64$ modes appeared to be sufficient to capture the essential dynamics of the flow. 


\section{Numerical results and discussion}

We begin by considering a region of parameter space where the bifurcation from the basic state is synchronous with the forcing and axisymmetric. We have selected to keep the forcing frequency $\omega_{f}=30$, the rotation Reynolds number $R e_{i}=200$, the gap $e=0.905$, the length to gap ratio $\Lambda=10$, and have varied the forcing amplitude $R e_{a}$. For the infinite case, $\Lambda \rightarrow \infty$, the Floquet analysis predicts a bifurcation to occur at $R e_{a} \approx 74$ with an axial wavenumber $k \approx 2.4$. In unforced Taylor-Couette flow, one would expect to fit four cells with $k=2.4$ into an $\Lambda=10$ annulus, but with the forcing, Ekman cells with aspect ratio about one are also formed at the endwalls, so that one ends up with about three Taylor cells in the forced system.

For $R e_{a}>75$, the system is "stable". By this we mean that apart from the regions near the endwalls where the Ekman cells are, the flow in the rest of the annulus is essentially independent of the axial direction. In the $\Lambda \rightarrow$ $\infty$ case, it is clear what is meant by a stable state; the basic state has no axial $(z)$ dependence and instability

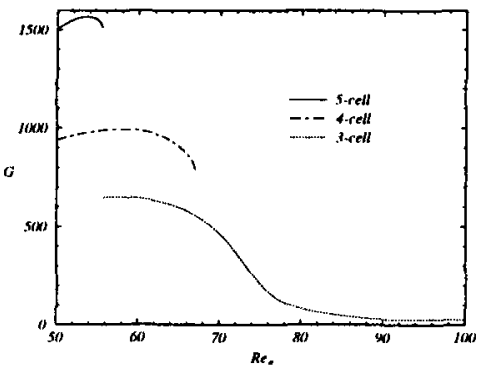

Fig. 1. $G$ versus $R e_{a} ; R e_{i}=200, \omega_{f}=30$, $e=0.905, \Lambda=10$.

is characterized by the onset of $z$-dependence. For finite $A$, the vortex lines are bent near the endwalls and converge to the singular corners where the stationary endwalls and the rotating and harmonically oscillating inner cylinder meet, thus producing axial variations in the flow for any finite $R e_{i}$ or $R e_{a}$. This leads to imperfect bifurcations in the finite $\Lambda$ cases. A analogous situation for the unforced Taylor-Couette flow in finite annulus has been discussed in detail by Benjamin (1978). As a measure of the flow state, we have selected the following indicator to measure the degree of axial dependence:

$$
G=\left(\max _{\text {period }}\left\|(\partial v / \partial z)^{2}\right\|-\min _{\text {period }}\left\|(\partial v / \partial z)^{2}\right\|\right),
$$

where $\|\cdot\|=\int_{\Lambda / 3}^{2 \Lambda / 3} \int_{0}^{1} \cdot d r d z$. Note that in the above definition, the axial dependence near the top and bottom wall is excluded in order to capture the main characteristics of the flow in the middle section of the cylinder.

Figure 1 shows $G$ versus $R e_{a}$. Note that there are three distinct branches. The branch labeled 3-cell has roughly three Taylor cells most of the time, branch 4-cell has four, and 5-cell has five. The 3-cell branch shows the typical form of an imperfect bifurcation, and the unfolding occurs in the vicinity of the perfect bifurcation of the $\Lambda \rightarrow \infty$ case, i.e. near $R e_{a}=74$. The 4cell branch could be interpreted as the "anomalous" branch (see Benjamin 
\& Mullin 1981), but such an interpretation requires further investigation of the continuation of the unstable part of the 4-cell branch around the turning point at $R e_{a} \approx 67$. The 3-cell branch losses stability for $R e_{a}<55.5$, and a 5 -cell branch exists for $R e_{a}<55.5$. A full investigation of the possible interconnections between these branches awaits the development of techniques to compute unstable periodic branch solutions for large periodically-forced systems.

All the solution branches depicted in figure 1 are synchronous with the forcing and preserve the half period translation/reflection symmetry. Keeping all parameters as in the previous parameter study, except for fixed $R e_{a}=80$ and variable $R e_{i}$ from $200 \mathrm{up}$, another slice of parameter space is investigated. For $R e_{i}<280$, the flow corresponds to the 3-cell branch, and is synchronous with the forcing. Due to the half period translation/reflection symmetry, the power spectral density (PSD) of $G$ over several forcing periods shows a signal at frequency $\omega=60$, and its higher harmonics, which is twice the forcing frequency $\omega_{f}=30$. At $R e_{i}=281$, the nonlinear evolution takes hundreds of periods for the transients to be damped following a step-change from $R e_{i}=280$ to 281 , and these transients are oscillatory, suggesting a pair of complex conjugated eigenvalues approaching the unit circle. The plots of the time-series and the power spectral density (PSD) of $G$ and the phase portrait $G(t)$ vs. $G(t+T / 8)$ for $R e_{i}=200$ and 280 shown in figure 2 indicate the simple limit cycle behavior.

At $R e_{i}=282$, the system settles down to a 2 -torus flow that has broken the half period translation/reflection symmetry, so not only is there symmetry-breaking and hence period-doubling, but there is also a Hopf bifurcation from a limit cycle to a 2 -torus. The power spectral density (PSD) of the $R e_{i}=200,281$, and 282 cases are shown in figures 2 . Note that the PSD of the $R e_{i}=282$ case has a signal at $\omega=30$, indicating the breaking of the symmetry and period-doubling, as well as a modulation frequency at $\omega \approx 3.33$ which is the second frequency on the 2-torus. The modulation is quite apparent from the corresponding time series, and the locking of the modulation frequency and the forcing frequeuncy in a 9:1 ratio is apparent from both the PSD and the phase portrait which shows 9 distinct loops. As $R e_{i}$ is increased further to 285 (see figure $2 d$ ), the flow on the 2-torus is no longer locked.

It should be noted that the onset of a Hopf bifurcation from a limit cycle to a 2-torus just described above has occurred in an axisymmetric system. This type of bifurcation in the $A \rightarrow \infty$ system has always been associated with the breaking of the azimuthal symmetry, but preserved the half period translation/reflection symmetry at onset. The presence of the endwalls in the finite $\Lambda$ case is responsible for the breaking of the half period translation/reflection symmetry and seems to be due to the nonlinear coupling between the Ekman vortices on the endwalls and the Taylor vortices in the interior. 
(a) $R e_{i}=200$
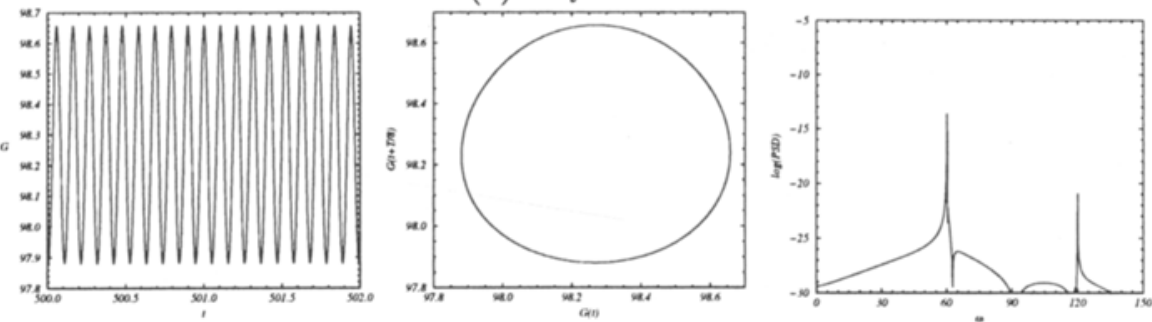

(b) $R e_{i}=281$
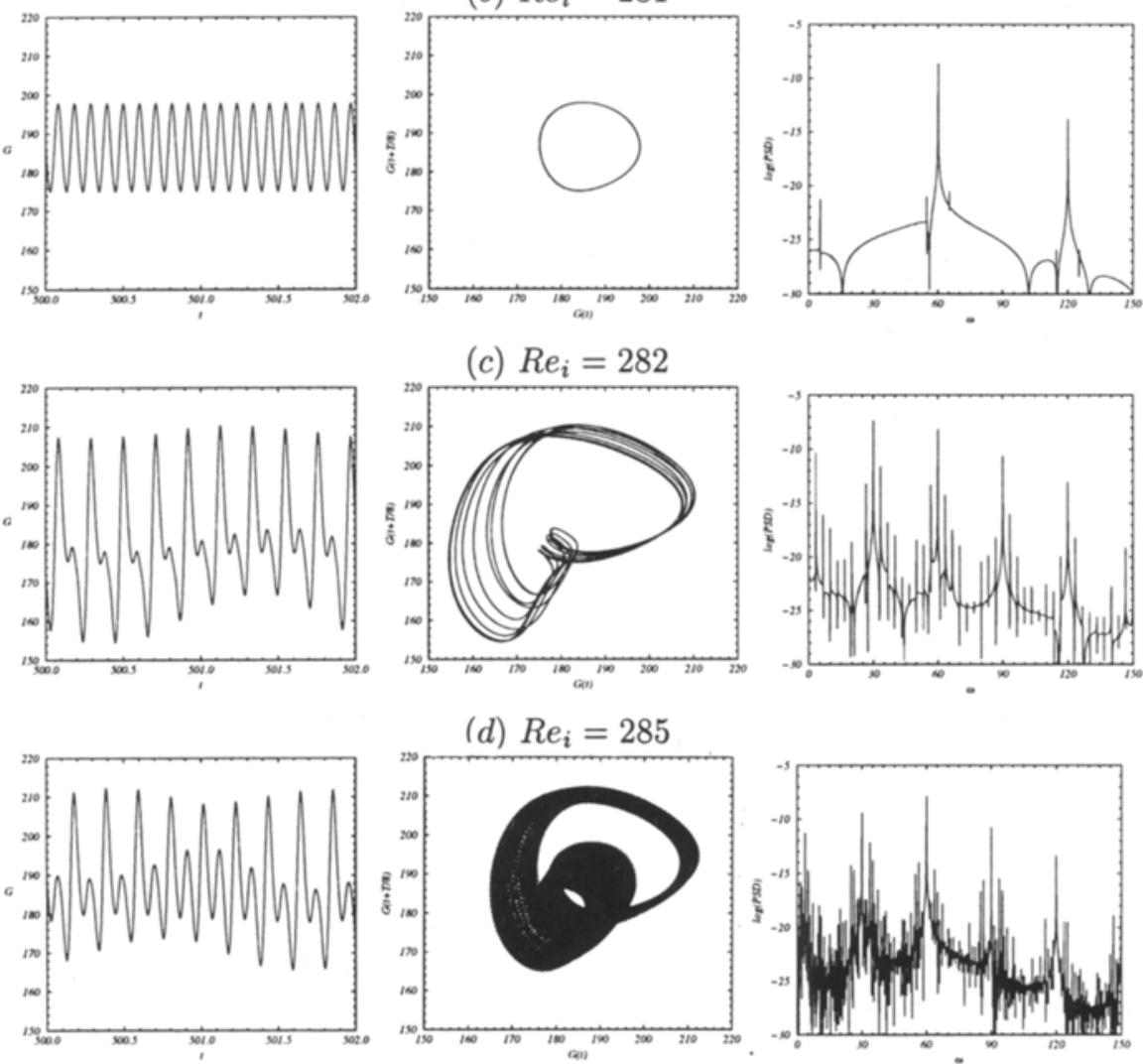

Fig. 2. Plots of (i) $G(t)$ over several periods of the forcing $T=2 \pi / \omega_{f}$, (ii) phase portrait of $G$ and its $T / 8$ delay, and (iii) power spectral density of $G(t)$ over $1252 T$, for $R e_{a}=80, \omega_{f}=30, e=0.905, \Lambda=10$, and $R e_{i}$ as indicated. 


\section{Summary}

For the physically realistic case of finite length concentric cylinders, the endwalls and the finite aspect ratio of the annulus are dynamically important. These nonlinear effects were investigated here with a very efficient and accurate spectral-projection method for solving the fully nonlinear axisymmetric Navier-Stokes equations. We have focused our attention on the axisymmetric states, guided by the results of the fully three-dimensional Floquet analysis, and considered two scenarios: varying respectively the forcing amplitude $R e_{a}$ and the Couette flow Reynolds number $R e_{i}$ while keeping other parameters fixed. In the former case, with $R e_{i}$ relatively low, the nonlinear response of the system was synchronous with the forcing frequency, and as $R e_{a}$ was reduced the flow underwent a smooth transition to a state with Taylor cells. As $R e_{a}$ was further reduced, additional solution branches, all synchronous with the forcing, were encountered. The existence of multiple flow states with various length scales is typical of pattern forming systems in enclosed regions. When $R e_{a}$ was fixed and $R e_{i}$ increased, we encountered a space-time symmetry breaking leading to flow on a 2-torus. Such a bifurcation is not predicted for the axisymmetric states from the Floquet analysis, and it appears that it is peculiar to the finite $A$ situation, with the coupling between the endwall Ekman vortices and the Taylor cells being responsible for the symmetry breaking.

\section{Acknowledgments}

This work was supported by NSF grants DMS-9512483 and DMS-9706951.

\section{References}

Benjamin, T. B., "Bifurcation phenomena in steady flows of a viscous fluid," Proc. R. Soc. Lond. A. 359, 1-26, 1978.

Benjamin, T. B. \& Mullin, T., "Anomalous modes in the Taylor experiment," Proc. R. Soc. Lond. A. 377, 221-249, 1981.

HU, H. C. \& KELlY, R. E., "Effect of a time-periodic axial shear flow upon the onset of Taylor vortices," Phys. Rev. E 51, 3242-3251, 1995.

Lopez, J. M. \& Shen, J., "An Efficient Spectral-Projection Method for the NavierStokes Equations in Cylindrical Geometries I. Axisymmetric cases," J. Comput. Phys. 139, 308-326, 1998.

MARques, F. \& LOPEZ, J. M., "Taylor-Couette flow with axial oscillations of the inner cylinder: Floquet analysis of the basic flow," J. Fluid Mech. 348, 153-175, 1997.

SHEN, J., "Efficient spectral-Galerkin method I. Direct solvers for second- and fourth-order equations by using Legendre polynomials," SIAM J. Sci. Comput. 15, 1489-1505, 1994.

Shen, J., "Efficient spectral-Galerkin methods III. Polar and cylindrical geometries," SIAM J. Sci. Comput. 18, 1583-1604, 1997.

WeIsBerg, A., Control of transition in Taylor-Couette flow with axial motion of the inner cylinder, Ph.D. Thesis, Princeton University, 1996.

Weisberg, A., Kevrekidis, I. G. \& Smits, A. J., "Delaying transition in TaylorCouette flow with axial motion of the inner cylinder," J. Fluid Mech. 348, 141-151, 1997. 